\title{
Ensinando Ciências Básicas através de Casos Clínicos: Percepção dos Estudantes de Fisiologia sobre o Uso deste Método
}

\author{
Teaching Basic Science By Clinical Cases: Perception Of Physiology Students About The Use Of This \\ Method
}

Ben-Hur Souto das Neves, Inaê de Oliveira Marcelo"; Matheus Henrique Naumann"; Pâmela Billig Mello-Carpes ${ }^{1 *}$,

${ }^{1}$ Grupo de Pesquisa em Fisiologia, Universidade Federal do Pampa

*e-mail: pamelacarpes@unipampa.edu.br

Support: Programa PDA, Universidade Federal do Pampa.

\begin{abstract}
Resumo
Métodos ativos de ensino têm sido adotados na formação de profissionais de diferentes áreas, estimulando o pensamento crítico e, dessa forma, favorecendo a tomada de decisão rápida e eficiente. O uso de casos clínicos em disciplinas da área da saúde é um exemplo. No entanto, a aplicação dessa ferramenta tem ocorrido principalmente em disciplinas clínicas. Neste contexto, propomos a utilização de casos clínicos no ensino de uma disciplina das ciências básicas: Fisiologia Humana. Os casos clínicos foram apresentados periodicamente, após aulas expositivas tradicionais, e uma discussão em grupo foi proposta para tentar entender a fisiologia envolvida em cada situação, aplicando o conhecimento teórico. As avaliações dos alunos mostraram que a atividade contribuiu para uma melhor compreensão do conteúdo, bem como para o desempenho dos alunos no curso de fisiologia.
\end{abstract}

Palavras-chave: casos clínicos; métodos ativos; fisiologia humana.

\begin{abstract}
Active teaching methods have been adopted in the training of professionals from different areas by stimulating critical thinking and, in this way, favoring quickly and efficient decision-making processes. The use of clinical cases in health area courses is an example. However, the application of this tool has been mainly in clinical courses. We propose the use of clinical cases in the teaching of a basic science course: Human Physiology. Clinical cases were presented periodically, after traditional classes, and a group discussion was proposed to try understanding the physiology behind each situation, applying the theoretical knowledge. The students' evaluations showed that the activity contributed to a better contents' understanding, as well as to the students' performance in Physiology course.
\end{abstract}

Keywords: clinical cases; active methods; human physiology. 
National award: Teaching Basic Science By Clinical Cases: Perception Of Physiology Students About The Use Of This Method

\section{Ficha da atividade/ material}

\begin{tabular}{|l|l|}
\hline Título & $\begin{array}{l}\text { Ensinando ciências básicas através de casos clínicos: Percepção dos } \\
\text { estudantes de Fisiologia sobre o uso deste método }\end{array}$ \\
\hline Categoria & Prática de ensino \\
\hline Tipo & $\begin{array}{l}\text { Atividades didáticas inovadora, com uso de casos clínicos no ensino de uma } \\
\text { ciência básica, a Fisiologia Humana. }\end{array}$ \\
\hline Público-alvo & $\begin{array}{l}\text { Alunos de Fisiologia Humana, do primeiro e segundo ano de graduação em } \\
\text { cursos da área da saúde }\end{array}$ \\
\hline $\begin{array}{l}\text { Conteúdos } \\
\text { abordados }\end{array}$ & $\begin{array}{l}\text { Fisiologia das células excitáveis (potenciais bioelétricos de membrana, } \\
\text { excitabilidade neural); Comunicação entre o Sistema Nervoso Central e o } \\
\text { Sistema Muscular; Contração muscular; Sistema Nervoso Central (controle } \\
\text { motor, processamento de informação sensorial, funções cognitivas, etc.); } \\
\text { Sistema Cardiovascular; e Sistema Respiratório. }\end{array}$ \\
\hline $\begin{array}{l}\text { Objetivos } \\
\text { educacionais }\end{array}$ & $\begin{array}{l}\text { Promover discussão sobre os conteúdos trabalhados em aula teórica e prática; } \\
\text { Associar com possíveis situações que os estudantes encontrarão em suas vidas } \\
\text { profissionais; Tornar os estudantes capazes de visualizar concretamente a } \\
\text { importância da fisiologia para a prática profissional. }\end{array}$ \\
\hline Duração & $\begin{array}{l}\text { Cada discussão de Caso Clínico (CC) durou aproximadamente 1h, sendo } \\
\text { dividido nos seguintes momentos: (i) apresentação do caso clínico pela } \\
\text { professora (cerca de 20 min.); (ii) discussão e identificação do problema em } \\
\text { grupo (cerca de 20 min.); e, (iii) discussão e proposição de soluções (de 15 - 20 } \\
\text { min.). }\end{array}$ \\
\hline Mriação & $\begin{array}{l}\text { Caso clínico elaborado com base em situações potencialmente reais, } \\
\text { apresentado por meio de Power Point, com dados de exames físico, } \\
\text { bioquímicos, de imagem, etc., conforme o caso específico. }\end{array}$ \\
\hline utilizados \\
$\begin{array}{l}\text { L1 de fevereiro de 2017. } \\
\text { A partir de 01 de março de 2017. }\end{array}$ \\
\hline NA
\end{tabular}


National award: Teaching Basic Science By Clinical Cases: Perception Of Physiology Students About The Use Of This Method

\section{Introdução}

O processo de ensino-aprendizagem é marcado por desafios tanto para o professor quanto para o aluno. Vários métodos para ajudar a superar esses desafios e melhorar a aprendizagem dos alunos têm sido propostos. Estudos anteriores mostraram que o engajamento de alunos com métodos alternativos de ensino-aprendizagem pode aumentar o interesse por conteúdos relacionados à biologia, além de contribuir para o processo de aprendizagem [1-3]. Estes diferentes métodos, que incluem o uso de jogos, atividades laboratoriais, sala de aula invertida, etc., que são diferentes do ensino tradicional, atraem a atenção dos alunos para o conteúdo e têm sido usados em diversas áreas [3-6], estimulando pensamento crítico e capacidade de julgar situações [7].

Durante a graduação, é essencial que os alunos desenvolvam o pensamento crítico, tornando-os capazes de tomar decisões rápidas [8]. O uso de diferentes métodos ativos nas ciências da saúde pode incentivar os alunos a adquirir habilidades específicas de resolução de problemas e a capacidade de aplicar seu conhecimento prévio [7, 9]. Métodos como a aprendizagem baseada em problemas (PBL - do inglês Problem Based Leraning) e a aprendizagem baseada em equipes (TBL - do inglês Team Based Learning) têm sido usados para encorajar o envolvimento do aluno, aprendizado autêntico, trabalho em equipe, habilidades de resolução de problemas e estudos interdisciplinares [9-11], características essenciais para a formação de um profissional de saúde. Esses métodos geralmente envolvem o uso de casos clínicos, mas requerem uma profunda reorganização curricular; no entanto, casos clínicos mais simples, não necessariamente no formato clássico do PBL, também podem ser associados à cursos desenvolvidos em modelos tradicionais.

Apesar do aumento relativo do uso de casos clínicos em ciências da saúde, eles são comumente usados no contexto de disciplinas clínicas, nos anos finais da graduação. Aqui, propomos o uso de casos clínicos em um curso tradicional de ciências básicas (fisiologia humana) com a intenção de promover uma discussão sobre os conteúdos trabalhados em sala de aula teórica e prática e associá-los a possíveis situações que os estudantes encontrarão posteriormente em sua vida profissional. Com essa prática, pretendemos permitir que os alunos visualizem concretamente a importância da fisiologia para a prática profissional, que muitas vezes é difícil e às vezes rara nos primeiros anos de graduação [12]. 
National award: Teaching Basic Science By Clinical Cases: Perception Of Physiology Students About The Use Of This Method

\section{Desenvolvimento}

Para esta atividade, casos clínicos (CC) abordando os conteúdos de Fisiologia Humana foram elaborados pela equipe proponente. Uma situação clínica fictícia, mas perfeitamente compatível com a realidade, foi usada em cada caso. Algumas informações básicas sobre cada CC foram apresentadas aos alunos, tais como: (a) características físicas do paciente: dados como altura, peso corporal, características corporais, etc.; (b) queixa principal do paciente; (c) principais resultados da anamnese: dados como histórico da doença atual, histórico médico, estilo de vida, etc.; (d) exame físico: dados que provavelmente seriam obtidos durante o exame físico do paciente; (e) exames complementares: resultados de exames de imagem (ressonância magnética nuclear, por exemplo), exames de sangue (glicose, lipídios, etc.), de acordo com o CC.

Após a apresentação do CC, os estudantes deveriam associar a situação com conteúdos previamente trabalhados, para tentar identificar qual era a alteração fisiológica. Propusemos um CC diferente para cada sistema corporal estudado em sala de aula teórica e prática de fisiologia (exemplos no Quadro 1).

Quadro 1. Exemplos de casos clínicos elaborados para a prática de ensino.

\begin{tabular}{|c|c|c|}
\hline $\begin{array}{l}\text { Sistema } \\
\text { corporal/ } \\
\text { Conteúdo de } \\
\text { fisiologia }\end{array}$ & Sumário do caso clínico & Objetivos pedagógicos \\
\hline $\begin{array}{l}\text { Fisiologia das } \\
\text { células } \\
\text { excitáveis } \\
\text { (potenciais } \\
\text { bioelétricos de } \\
\text { membrana, } \\
\text { excitabilidade } \\
\text { neural) }\end{array}$ & $\begin{array}{l}\text { Paciente de } 43 \text { anos, sem história de doença } \\
\text { crônica, não fumante. Paciente referiu perda de } \\
\text { visão no olho direito, parestesia no membro superior } \\
\text { direito, dormência em ambos os membros inferiores } \\
\text { e dificuldade para deambular. A investigação do } \\
\text { histórico médico revelou que o paciente já } \\
\text { apresentava uma condição não recorrente } \\
\text { semelhante há cerca de } 8 \text { anos. Frequência } \\
\text { cardíaca e pressão arterial normais. A ressonância } \\
\text { magnética nuclear demonstra um foco múltiplo de } \\
\text { desmielinização na substância branca } \\
\text { periventricular e subcortical. }\end{array}$ & $\begin{array}{l}\text { Os estudantes deveriam } \\
\text { relacionar a destruição da } \\
\text { mielina com os sintomas dos } \\
\text { pacientes, considerando o } \\
\text { papel da mielina na } \\
\text { transmissão do potencial de } \\
\text { ação ao longo do axônio. O } \\
\text { paciente do caso tinha } \\
\text { Esclerose Múltipla, mas não } \\
\text { era necessário que os alunos } \\
\text { dessem um diagnóstico } \\
\text { específico. }\end{array}$ \\
\hline $\begin{array}{l}\text { Comunicação } \\
\text { entre o sistema } \\
\text { nervoso e os } \\
\text { músculos, e } \\
\text { contração } \\
\text { muscular. }\end{array}$ & $\begin{array}{l}\text { Foi apresentado um caso de uma paciente de } 38 \\
\text { anos de idade, uma mulher sem história de doença } \\
\text { crônica, não fumante. Em uma consulta médica de } \\
\text { rotina ela relatou fraqueza muscular, falta de ar e } \\
\text { dificuldade para engolir. O médico realizou o exame } \\
\text { físico e solicitou alguns exames adicionais para } \\
\text { confirmar o diagnóstico. Sinais vitais normais. } \\
\text { Exame físico: ptose palpebral e voz anasalada; } \\
\text { exame neurológico indica resposta sensorial } \\
\text { normal; e respostas reflexas normais, embora uma } \\
\text { pequena fraqueza muscular esteja presente. } \\
\text { Eletroneuromiografia - Repetição do teste de } \\
\text { estimulação do nervo no bíceps: A resposta ao } \\
\text { quinto estímulo no trem de estímulo diminuiu em } \\
20 \% \text {. Teste plasmático: presença de anticorpos }\end{array}$ & $\begin{array}{l}\text { Os estudantes deveriam } \\
\text { relacionar a destruição dos } \\
\text { receptores colinérgicos com a } \\
\text { fraqueza muscular do } \\
\text { paciente, considerando o } \\
\text { papel dos receptores } \\
\text { colinérgicos na excitação- } \\
\text { contração muscular. O } \\
\text { paciente do caso tinha } \\
\text { Miastenia Gravis, mas não era } \\
\text { necessário que os alunos } \\
\text { dessem um diagnóstico } \\
\text { específico. }\end{array}$ \\
\hline
\end{tabular}




\begin{tabular}{|c|c|c|}
\hline & $\begin{array}{l}\text { dirigidos contra o receptor de acetilcolina: } 0,44 \\
\mathrm{nmol} / \mathrm{L} \text { (valor normal }<0,20 \mathrm{nmol} / \mathrm{L} \text { ). }\end{array}$ & \\
\hline $\begin{array}{l}\text { Fisiologia do } \\
\text { Sistema } \\
\text { Nervoso Central } \\
\text { (controle motor, } \\
\text { processamento } \\
\text { de informação } \\
\text { sensorial, } \\
\text { funções } \\
\text { cognitivas, etc.) }\end{array}$ & $\begin{array}{l}\text { Foi apresentado um caso de paciente do sexo } \\
\text { masculino, } 62 \text { anos de idade. Consultado com um } \\
\text { médico após dificuldade progressiva na execução } \\
\text { de tarefas manuais, como abotoar e pentear, e } \\
\text { dificuldades de marcha. O paciente refere que teve } \\
\text { uma pedra nos rins há } 12 \text { anos, mas não } \\
\text { apresentou nenhum outro problema de saúde } \\
\text { significativo ao longo da vida. Sinais vitais e SaO2 } \\
\text { normais. Exame do aparelho locomotor: durante o } \\
\text { movimento passivo, verifica-se que os músculos } \\
\text { são rígidos, provocando o movimento da "roda } \\
\text { dentada" durante o alongamento. Ao caminhar, o } \\
\text { paciente tem dificuldade em dar o primeiro passo, } \\
\text { mas consegue caminhar suavemente com uma } \\
\text { marcha arrastada. Um tremor em repouso da mão } \\
\text { esquerda que diminui ao executar uma tarefa é } \\
\text { observado. }\end{array}$ & $\begin{array}{l}\text { Os estudantes deveriam } \\
\text { relacionar os sintomas com a } \\
\text { doença de Parkinson, que } \\
\text { envolve a destruição dos } \\
\text { neurônios dopaminérgicos da } \\
\text { substância negra, envolvidos } \\
\text { no controle e coordenação dos } \\
\text { movimentos voluntários. }\end{array}$ \\
\hline $\begin{array}{l}\text { Sistema } \\
\text { cardiovascular }\end{array}$ & $\begin{array}{l}\text { Foi apresentado um caso de paciente do sexo } \\
\text { masculino, } 37 \text { anos, sem história prévia de } \\
\text { hipertensão. O paciente procurou o médico com } \\
\text { pressão alta. O homem relatou que não usava } \\
\text { drogas ou medicamentos, exceto pelos cigarros. Ele } \\
\text { tinha uma dieta "normal" (sem cuidados especiais), } \\
\text { embora ele não abrisse mão de um bom churrasco } \\
\text { de "carne gorda" em todos os finais de semana. } \\
\text { Percebe-se que o homem está acima do peso ideal. } \\
\text { Pressão sanguínea medida: } 180 / 110 \text { mmHg. Para } \\
\text { entender o caso clínico, exames de sangue foram } \\
\text { realizados. Testes mostraram que o homem tinha } \\
\text { elevada concentração de renina no sangue. } \\
\text { Exames de imagem mostraram uma estenose } \\
\text { grande que quase bloqueava o fluxo sanguíneo } \\
\text { através das artérias renais. }\end{array}$ & $\begin{array}{l}\text { Este caso abordou o papel do } \\
\text { sistema renina-angiotensina- } \\
\text { aldosterona no controle da } \\
\text { pressão arterial. Os } \\
\text { estudantes tiveram que } \\
\text { relacionar hipertensão arterial } \\
\text { sistêmica com aumento dos } \\
\text { níveis de renina no sangue, } \\
\text { considerando o papel da } \\
\text { renina no controle da pressão } \\
\text { arterial e os mecanismos } \\
\text { fisiológicos envolvidos na sua } \\
\text { liberação - eles deveriam } \\
\text { entender que a renina é } \\
\text { elevada considerando o } \\
\text { bloqueio do fluxo sanguíneo } \\
\text { nas artérias renais. }\end{array}$ \\
\hline $\begin{array}{l}\text { Sistema } \\
\text { respiratório }\end{array}$ & $\begin{array}{l}\text { Foi apresentado um caso de um paciente do sexo } \\
\text { masculino de } 55 \text { anos de idade. Ele se queixava de } \\
\text { fadiga e falta de ar persistente, que piorava durante } \\
\text { o exercício. Paciente trabalhou por } 20 \text { anos em uma } \\
\text { fábrica de automóveis e foi demitido há } 5 \text { anos. } \\
\text { Além de ter um histórico médico de infecções } \\
\text { respiratórias, o paciente fumava cigarros desde a } \\
\text { adolescência. Atualmente, ele estima fumar um } \\
\text { maço de cigarros por dia. Ele apresenta a tosse } \\
\text { crônica, que é pior de manhã. Exame físico: Sinais } \\
\text { vitais normais. Ausculta pulmonar: O paciente está } \\
\text { com desconforto respiratório leve, com uma alta } \\
\text { taxa respiratória e respiração superficial. A sibilância } \\
\text { expiratória final é ouvida na ausculta. Exceto por } \\
\text { isso, seus pulmões parecem normais à ausculta e à } \\
\text { percussão. O teste de função pulmonar revelou: } \\
\text { VEF1 }=70 \% \text { do previsto. Pico expiratório = } 60 \% \text { do } \\
\text { previsto; capacidade vital forçada = } 90 \% \text { do } \\
\text { previsto. } \mathrm{Na} \text { radiografia pulmonar: aparentemente } \\
\text { normal, exceto pela hiperinsuflação. Na gasometria } \\
\text { arterial: } \mathrm{PaO}_{2}=75 \mathrm{mmHg} \mathrm{PaCO}_{2}=42 \mathrm{mmHg} \text { mH }= \\
7,32 \text {. }\end{array}$ & $\begin{array}{l}\text { O paciente tinha uma doença } \\
\text { pulmonar obstrutiva crônica. } \\
\text { Os estudantes deveriam } \\
\text { relacionar a obstrução das } \\
\text { vias aéreas e a destruição } \\
\text { alveolar com a falta de ar, } \\
\text { considerando a redução das } \\
\text { trocas gasosas e do fluxo } \\
\text { respiratório. }\end{array}$ \\
\hline
\end{tabular}


National award: Teaching Basic Science By Clinical Cases: Perception Of Physiology Students About The Use Of This Method

\section{Procedimento de uso do material ou aplicação da atividade}

\subsection{Apresentação dos casos clínicos pelo/a professor/a}

Sugerimos que inicialmente os alunos sejam divididos em equipes de 5-7 participantes, para facilitar as discussões nas etapas subsequentes. Em seguida, o/a professor/a deve fazer a apresentação do caso clínico (CC). Isto pode ser feito por meio de uma apresentação de slides, ou com fichas e exames fictícios entregues a cada grupo.

\subsection{Discussão e identificação do problema}

Após a apresentação do CC, os grupos de alunos tem cerca de 20 minutos para discutir o caso e apresentar evidências que possam auxiliar na definição de um possível problema (a ideia é tentar identificar qual a função fisiológica que está alterada, e não necessariamente definir um diagnóstico, até porque trata-se de uma disciplina básica). Durante a atividade os alunos podem discutir e consultar bibliografia. Imediatamente após o término das discussões entre os alunos, cada grupo deve apresentar as conclusões da equipe. Então, juntos, a partir dos conteúdos trabalhados anteriormente em sala de aula teórica e prática e das discussões, os alunos de todos os grupos debatem e chegam a um consenso sobre as mudanças observadas.

\subsection{Discussão e proposta de soluções}

Uma vez que as alterações fisiopatológicas observadas no paciente foram discutidas, a equipe de trabalho deve propor alternativas de intervenção, práticas que a equipe acredita que possam evitar, minimizar e/ou reverter os problemas detectados na situação fictícia. Considerando que os alunos estão no primeiro e no segundo ano de graduação, não se espera que eles possam propor um plano de tratamento, mas que identifiquem em quais aspectos o tratamento deveria se concentrar. Mais uma vez, cada grupo deve apresentar as conclusões da equipe e, em seguida, os alunos de todos os grupos debatem e chegam a um consenso. Esta parte da atividade requer aproximadamente mais de 15 a 20 minutos.

\section{Procedimentos de avaliação}

Desenvolvemos esta prática de ensino com 99 alunos matriculados em cursos de Fisiologia Humana, ministrados no primeiro e segundo ano de graduação em Enfermagem e Fisioterapia na Universidade Federal do Pampa, no campus Uruguaiana, 
National award: Teaching Basic Science By Clinical Cases: Perception Of Physiology Students About The Use Of This Method

no Rio Grande do Sul (Brasil). Todas as atividades foram aprovadas pela Comissão de Ensino Institucional Local (Comitê de Revisão Institucional $n^{\circ}$ 10.006.16) e foram realizadas no primeiro semestre acadêmico de 2017.

Ao final da atividade, os alunos foram convidados a responder um formulário de avaliação sobre a contribuição da atividade para a sua compreensão dos conteúdos curriculares. Com exceção das três primeiras e da última questão do formulário, estas foram apresentadas no formato da escala Likert [13], composto por afirmações para os quais os alunos deveriam indicar seu grau de concordância (Quadro 2).

Quadro 2 Perguntas utilizadas na avaliação da atividade e os resultados da percepção dos alunos.

\begin{tabular}{|c|c|}
\hline Questões & Opções de resposta \\
\hline A. Como você avalia seu desempenho na & ( ) Ótimo \\
\hline disciplina de fisiologia até agora? & ( ) Bom \\
\hline & ( ) Regular \\
\hline & ( ) Ruim \\
\hline $\begin{array}{l}\text { B. Você acredita que o uso de casos clíni- } \\
\text { cos em nossas aulas contribuiu para o }\end{array}$ & ( ) Sim \\
\hline $\begin{array}{l}\text { seu desempenho em outros disciplinas } \\
\text { além da fisiologia? }\end{array}$ & ( ) Não \\
\hline C. Você recomendaria que outros professo- & ( ) Sim \\
\hline res utilizem casos clínicos em suas sa- & ( ) Não \\
\hline las de aula? & ( ) Não tenho certeza \\
\hline D. O uso de casos clínicos colaborou com & ( ) Eu concordo totalmente com esta afirmação. \\
\hline o seu desempenho em Fisiologia Huma- & ( ) Eu concordo parcialmente com esta afirmação. \\
\hline & ( ) Não concordo nem discordo \\
\hline & ( ) Eu discordo parcialmente desta afirmação. \\
\hline & ( ) Eu discordo totalmente dessa afirmação. \\
\hline E. O uso de casos clínicos contribuiu para & ( ) Eu concordo totalmente com esta afirmação. \\
\hline o seu entendimento dos conteúdos de & ( ) Eu concordo parcialmente com esta afirmação. \\
\hline & ( ) Não concordo nem discordo \\
\hline & ( ) Eu discordo parcialmente desta afirmação. \\
\hline & ( ) Eu discordo totalmente dessa afirmação. \\
\hline F. Casos clínicos são ferramentas interes- & ( ) Eu concordo totalmente com esta afirmação. \\
\hline santes para o ensino de fisiologia & () Eu concordo parcialmente com esta afirmação. \\
\hline & ( ) Não concordo nem discordo \\
\hline & ( ) Eu discordo parcialmente desta afirmação. \\
\hline & ( ) Eu discordo totalmente dessa afirmação. \\
\hline G. Os casos clínicos permitiram a aproxi- & ( ) Eu concordo totalmente com esta afirmação. \\
\hline mação dos conteúdos de fisiologia com & ( ) Eu concordo parcialmente com esta afirmação. \\
\hline & ( ) Não concordo nem discordo \\
\hline & ( ) Eu discordo parcialmente desta afirmação. \\
\hline & ( ) Eu discordo totalmente dessa afirmação. \\
\hline H. O uso de casos clínicos deu mais senti- & ( ) Eu concordo totalmente com esta afirmação. \\
\hline do aos conteúdos e permitiu maior apli- & ( ) Eu concordo parcialmente com esta afirmação. \\
\hline cabilidade dos mesmos. & ( ) Não concordo nem discordo \\
\hline & ( ) Eu discordo parcialmente desta afirmação. \\
\hline & ( ) Eu discordo totalmente dessa afirmação. \\
\hline
\end{tabular}


National award: Teaching Basic Science By Clinical Cases: Perception Of Physiology Students About The Use Of This Method

\begin{tabular}{|l|l|}
\hline \multirow{1}{*}{$\begin{array}{l}\text { Na sua opinião, usar casos clínicos em } \\
\text { çães quiser) }\end{array}$} & $\begin{array}{l}\text { ( ) Importante para sua compreensão dos conceitos de } \\
\text { fisiologia, complementando as aulas teóricas. }\end{array}$ \\
\cline { 2 - 2 } & $\begin{array}{l}\text { ( ) Essencial para sua compreensão em fisiologia, pois } \\
\text { somente com as aulas não conseguiria acompanhar o } \\
\text { conteúdo. }\end{array}$ \\
\cline { 2 - 2 } & $\begin{array}{l}\text { ( ) Divertido, porque proporcionou um momento informal } \\
\text { de discussão coletiva dos conteúdos nos grupos. }\end{array}$ \\
\cline { 2 - 2 } & $\begin{array}{l}\text { ( ) Motivacional, porque me permitiu visualizar a } \\
\text { importância da fisiologia para o desempenho profissional. }\end{array}$ \\
\cline { 2 - 2 } & ( ) Desnecessário. \\
\hline
\end{tabular}

\section{Potencial educacional}

Considerando os resultados da prática de ensino proposta, apresentados a seguir, evidencia-se o potencial educacional de seu uso. Neste trabalho avaliamos o uso de CC adaptados para utilização na disciplina de Fisiologia Humana, mas casos deste tipo podem ser facilmente adaptados para utilização em diferentes ciências básicas, tais como histologia, bioquímica, biologia molecular, imunologia, entre outras. O uso de CC permite que os estudantes da área da saúde visualizem a importância destas disciplinas básicas para a prática profissional futura, algo que muitas vezes é abstrato aos estudantes. Entender a importância da disciplina e o significado dos conteúdos estudados é fundamental para motivar os estudantes a se engajarem nas atividades propostas.

\section{Diferencial com as atividades/materiais preexistentes}

Apesar o uso de CC no ensino universitário não ser por si só uma novidade, os CC geralmente são utilizados em disciplinas clínicas, ou em cursos cujo currículo propõe abordagens como o PBL ou TBL. Aqui, propomos o uso de CC adaptados para um curso de ciências básicas de cursos com currículo tradicional, com a intenção de promover uma discussão sobre os conteúdos estudados, relacionando-os com situações que os estudantes poderão encontrar na vida profissional. Esta prática permite que os alunos visualizem a importância da referida ciência básica para a prática profissional.

\section{Resultados da avaliação}

Um total de 99 alunos matriculados nos cursos de Fisiologia Humana responderam ao questionário; sendo 50 do curso de Enfermagem e 49 do curso de Fisioterapia. A média de idade dos estudantes foi de 22,15 anos.

Quando questionados sobre seu desempenho no curso de Fisiologia Humana, $7,7 \%(n=7)$ dos estudantes avaliou como ótimo; 56,56\% ( $n=56)$ como bom; 33,33\% ( $n=$ 33) como regular; e 3,3\% $(n=3)$ como ruim [Fig. 1A]. Questionados se o uso do CC durante as aulas contribuiu para a sua atuação em outros cursos além da fisiologia, 
National award: Teaching Basic Science By Clinical Cases: Perception Of Physiology Students About The Use Of This Method

$85,85 \%(n=85)$ afirmou que ele contribuiu; e 14,14\% $(n=14)$ que não [Fig 1B]. Além disso, quando perguntamos se recomendariam o uso de casos clínicos a outros professores, $89,89 \%(n=89)$ afirmou que sim [Fig. 1C].

A

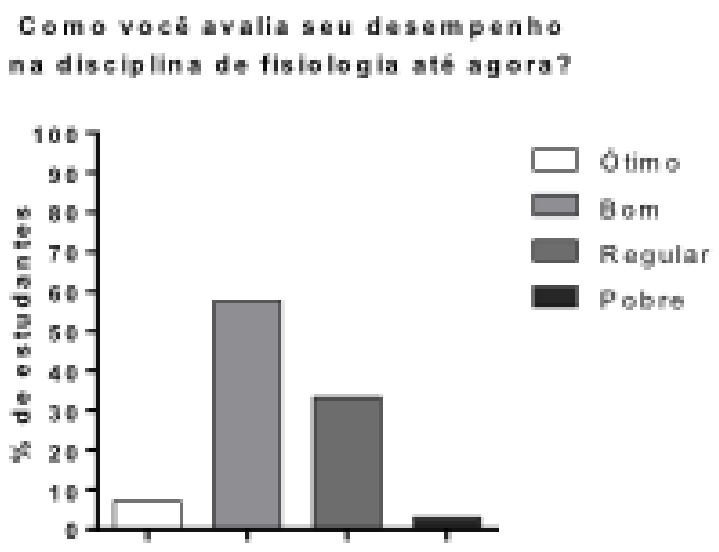

C

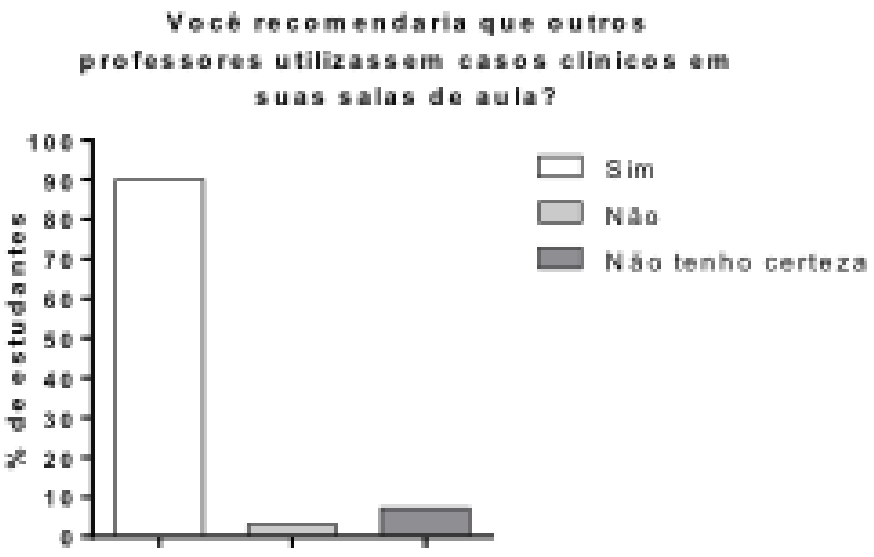

B

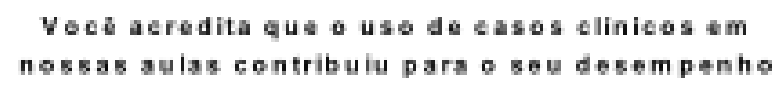
em outras diseiplinas alem da fisiologia?

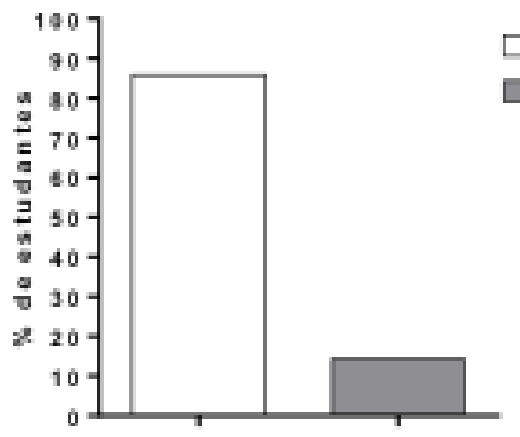

Figura 1. Percepção dos estudantes sobre: A. O seu desempenho no curso da fisiologia humana; B. O impacto do uso de casos clínicos em seu desempenho no curso de fisiologia humana; e, C. A recomendação do uso de casos clínicos em outros cursos.

A maioria dos estudantes $(76,76 \%, n=76)$ concordou plenamente com a afirmação de que o uso de casos clínicos colaborou com seu desempenho no curso de Fisiologia Humana, 19,19\% ( $n=19)$ concordou parcialmente com essa afirmação [Fig. 2A]. Além disso, $81,81 \%(n=81)$ concordou plenamente com a afirmação de que o uso de casos clínicos contribuiu para a compreensão dos conteúdos fisiológicos, $17,17 \%$ ( $n=17$ ) concordou parcialmente [Fig. 2B]. Ainda, $90,90 \%$ dos estudantes $(n=90)$ concordou plenamente com a afirmação de que o uso de casos clínicos é uma ferramenta interessante para o ensino de fisiologia; e 9,9\% $(n=9)$ concordou parcialmente com esta afirmação [Fig. 2C]. 
National award: Teaching Basic Science By Clinical Cases: Perception Of Physiology Students About The Use Of This Method

A

O uso de cases clinisos colabereu $50 \mathrm{~m}$ o seu desempenho em Fisiologila $\mathrm{Hum}$ ana

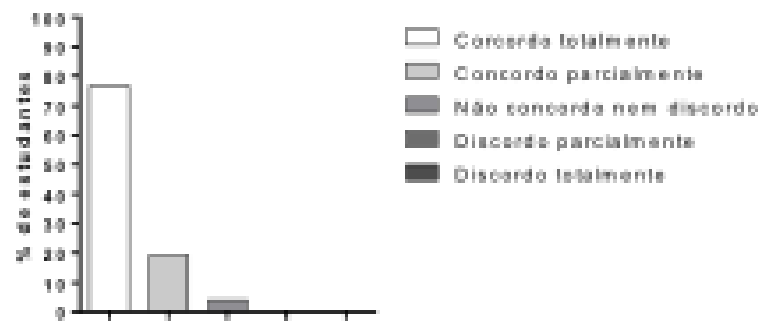

C

Casos elinicos abo ferramentus interessuntes para o ensino de Fisiolegia Humana

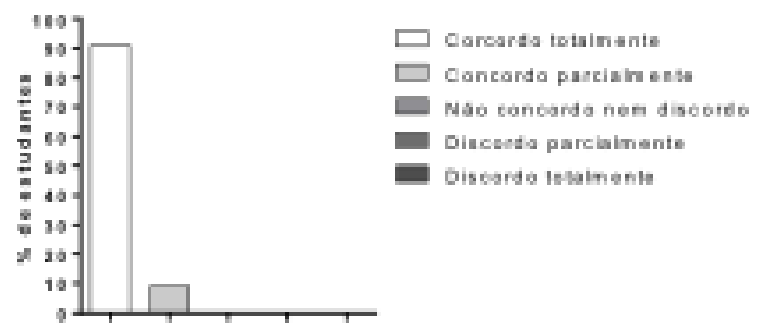

E

Ouso de casos clinicos foz m ais sentido permitiu maior aplieabilidade dos conteudos estudados em fisiologia

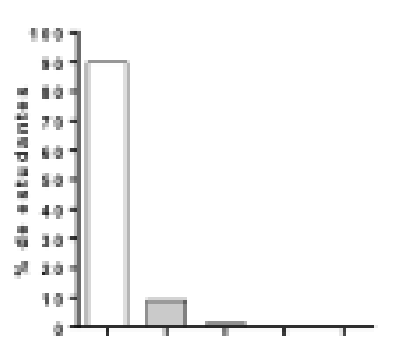

B o wso de casos clinices contribuie para 0 entendim ente des conteudos de Fislologia Hum ana

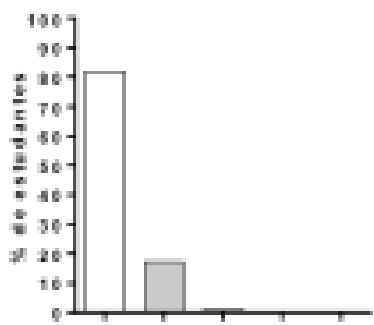

D Os casos clinices permitiram a aproximaşo dos contedes de fisiologia cem a pratica clinica

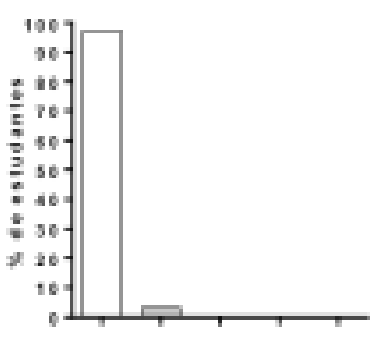

$\square$ Carsords totain exate $\square$ Cancanta parciainente $\square$ Nao canenes narn Estordo Diacordo aarsiaineate Disoseto tolainante

$\mathbf{F}$ Na sua epinibe, usaf casos clinices na sala de aula foi... (marque quantas opçoses quiser)

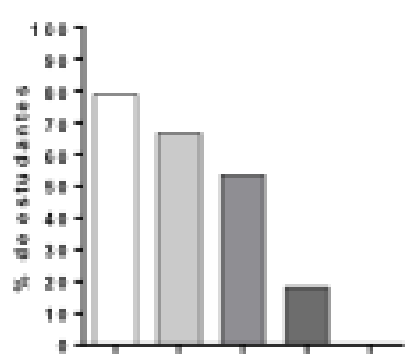

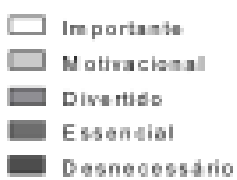

Figura 2. Percepções dos estudantes sobre o uso de casos clínicos no ensino e aprendizagem da fisiologia humana.

Considerando a afirmação de que o uso de casos clínicos em sala de aula permitiu uma aproximação dos conteúdos da fisiologia com a prática clínica, 96,7\% ( $n=96)$ concordou totalmente e 3,3\% ( $n=3)$ concordou parcialmente [Fig. 2D]. Por fim, quando afirmamos que o uso de casos clínicos deu mais sentido aos conteúdos estudados e permitiu uma maior aplicabilidade dos mesmos, $89,89 \%$ dos alunos $(n=89)$ concordou totalmente, 9,9\% $(n=9)$ concordou parcialmente [Fig . 2E].

$\mathrm{Na}$ última pergunta, pedimos aos alunos que opinassem sobre o uso de casos 
National award: Teaching Basic Science By Clinical Cases: Perception Of Physiology Students About The Use Of This Method

clínicos, considerando a experiência que tinham. Entre os respondentes, 78,78\% ( $n=78$ ) considerou importante o uso do CC para compreender os conceitos de fisiologia, complementando as aulas teóricas; $66,66 \%(n=66)$ considerou a atividade motivacional, pois permitiu visualizar a importância da Fisiologia para o seu desempenho profissional futuro; $53,53 \%$ ( $n=53$ ) achou divertido já que proporcionou um momento informal de discussão coletiva do conteúdo; e $18,18 \%(n=18)$ considerou essencial para sua compreensão da fisiologia, pois somente com as aulas não conseguiriam acompanhar o conteúdo [Fig. 2F].

\section{Considerações finais}

O conjunto de resultados da avaliação da prática de ensino demonstra que o uso de CC pode ser uma alternativa interessante para melhorar a qualidade dos processos de ensino e aprendizagem da fisiologia, de acordo com a percepção dos alunos envolvidos. Acreditamos que esta metodologia poderia ser facilmente adaptadas para utilização em outros cursos de ciências básicas, trazendo melhorias semelhantes no processo de ensino e aprendizagem.

Estudos anteriores mostram que $\mathrm{o}$ uso de metodologias ativas melhorou $\mathrm{o}$ desempenho dos alunos [14] e que esse tipo de método de ensino promove resultados positivos no ensino de fisiologia [3]. Aqui, todos os alunos consideram o uso do CC como uma ferramenta interessante para o ensino de fisiologia. A opinião geral dos estudantes sobre CC foi positiva, uma vez que atribuem características como "importante", "essencial", "divertida" e "motivadora" à atividade. Para o professor/a é importante que seus alunos aprendam e compreendam a aplicabilidade do conteúdo ensinado. Dessa forma, as escolhas metodológicas são uma importante responsabilidade do professor, que deve se tornar facilitador para o entendimento do aluno [15]. Além disso, se os professores podem tornar o aprendizado divertido e motivador, isso pode aumentar o interesse do aluno e melhorar sua disposição em aprender [16].

Outro ponto importante a considerar é que, durante sua futura carreira profissional, os alunos terão que trabalhar em grupo. Começar a prepará-los para promover atividades colaborativas em grupo nas aulas é fundamental. Aqui, os CCs foram discutidos em pequenos grupos primeiro e depois com toda a turma. Aparentemente, este método foi eficaz para desenvolver habilidades, desenvolvendo a comunicação e promovendo nos alunos a crença de que o trabalho em grupo contribui para habilidades importantes para a sua prática profissional [17]. 
National award: Teaching Basic Science By Clinical Cases: Perception Of Physiology Students About The Use Of This Method

\section{Referências}

[1] Bailey CM, Hsu CT, DiCarlo SE. Educational puzzles for understanding gastrointestinal physiology. The American journal of physiology. 1999;276(6 Pt 2):S1-18. Epub 2005/10/08.

[2] Neves B-HS, Altermann C, Gonçalves R, Lara MV, Mello-Carpes PB. Home-based vs. laboratory-based practical activities in the learning of human physiology: the perception of students. Advances in physiology education. 2017;41(1):89-93.

[3] Machado RS, Oliveira I, Ferreira I, das Neves BS, Mello-Carpes PB. The membrane potential puzzle: a new educational game to use in physiology teaching. Advances in physiology education. 2018;42(1):79-83. Epub 2018/01/18.

[4] Tune JD, Sturek M, Basile DP. Flipped classroom model improves graduate student performance in cardiovascular, respiratory, and renal physiology. Advances in physiology education. 2013;37(4):316-20.

[5] Altermann C, Gonçalves R, Lara MVS, Neves B-HS, Mello-Carpes PB. Observing and understanding arterial and venous circulation differences in a physiology laboratory activity. Advances in physiology education. 2015;39(4):405-10.

[6] Cagiltay NE, Ozcelik E, Ozcelik NS. The effect of competition on learning in games. Computers \& Education. 2015;87:35-41.

[7] Gurpinar E, Kulac E, Tetik C, Akdogan I, Mamakli S. Do learning approaches of medical students affect their satisfaction with problem-based learning? Advances in physiology education. 2013;37(1):85-8. Epub 2013/03/09.

[8] Weast D. Alternative teaching strategies: The case for critical thinking. Teaching Sociology. 1996;24(2):189-94.

[9] Kibble JD, Bellew C, Asmar A, Barkley L. Team-based learning in large enrollment classes. Advances in physiology education. 2016;40(4):435-42.

[10] Klegeris A, Hurren H. Impact of problem-based learning in a large classroom setting: student perception and problem-solving skills. Advances in physiology education. 2011;35(4):408-15. Epub 2011/12/06.

[11] Alimoglu MK, Yardım S, Uysal H. The effectiveness of TBL with real patients in neurology education in terms of knowledge retention, in-class engagement, and learner reactions. Advances in physiology education. 2017;41(1):38-43.

[12] Borges S, Mello-Carpes PB. Physiology applied to everyday: the practice of professional contextualization of physiology concepts as a way of facilitating learning. Advances in physiology education. 2014;38(1):93-5. Epub 2014/03/04.

[13] Sullivan GM, Artino AR, Jr. Analyzing and interpreting data from likert-type scales. Journal of graduate medical education. 2013;5(4):541-2.

[14] Rao SP, DiCarlo SE. Active learning of respiratory physiology improves performance on respiratory physiology examinations. Advances in physiology education. 2001;25(1-4):127-33.

[15] Akers TK. The physiology teacher, a facilitator of learning at all levels. The American journal of physiology. 1991;260(6 Pt 3):S32-3.

[16] DiCarlo SE. Too much content, not enough thinking, and too little fun! Advances in physiology education. 2009;33(4):257-64.

[17] Koh GC, Khoo HE, Wong ML, Koh D. The effects of problem-based learning during medical school on physician competency: a systematic review. CMAJ. 2008;178(1):34-41. 
National award: Teaching Basic Science By Clinical Cases: Perception Of Physiology Students About The Use Of This Method

\section{Agradecimentos}

Os autores agradecem a todos os estudantes de graduação que participaram das atividades propostas, assim como a Coordenação Acadêmica do campus Uruguaiana e a Pró-reitoria de Graduação da Universidade Federal do Pampa, que apoiaram a presente proposta. 